\title{
Molecular dynamics simulations of momentum and thermal diffusion properties of near-critical argon along isobars
}

\author{
Jakler Nichele ${ }^{\mathrm{a}, \mathrm{b}}$, Itamar Borges Jr. ${ }^{\mathrm{a}, \mathrm{b}}$, Alan B. Oliveira ${ }^{\mathrm{c}}$, Leonardo S. Alves ${ }^{\mathrm{a}, \mathrm{d}, *}$ \\ a Defense Engineering Graduate Program, Military Institute of Engineering, 22290-270 Rio de Janeiro, Brazil \\ b Chemical Engineering Department, Military Institute of Engineering, 22290-270 Rio de Janeiro, Brazil \\ ${ }^{c}$ Department of Physics, Federal University of Ouro Preto, 35400-00 Ouro Preto, Brazil \\ d Theoretical and Applied Mechanics Laboratory, Mechanical Engineering Department, Fluminense Federal University, $24210-240$ Niterói, Brazil
}

\section{A R T I C L E I N F O}

\section{Article history:}

Received 18 October 2015

Received in revised form 11 April 2016

Accepted 11 April 2016

Available online 12 April 2016

\section{Keywords:}

Shear viscosity

Bulk viscosity

Thermal conductivity

Equilibrium molecular dynamics

Stokes hypothesis

\begin{abstract}
A B S T R A C T
Three basic diffusion properties of argon - shear viscosity, bulk viscosity and thermal conductivity - were studied in the neighborhood of the critical point using molecular dynamics (MD) and the Lennard-Jones potential energy function. MD simulations were performed along the $1.0 P_{c}$ and $1.2 P_{c}$ isobars. Green-Kubo relations and a Lennard-Jones pair potential were used. Four different sets of Lennard-Jones parameters were used. A comparison of computed shear viscosity and thermal conductivity values with data available from the National Institute of Standards and Technology (NIST) displayed a good agreement. Results for bulk viscosity indicated that values of this property cannot be neglected in this thermodynamic region, a result that violates the traditional and much-assumed Stokes hypothesis in classical fluid mechanics. Furthermore, it was shown that in the neighborhood of the critical region the bulk viscosity can have larger values than the shear viscosity.
\end{abstract}

(c) 2016 Elsevier B.V. All rights reserved.

\section{Introduction}

Fluid dynamics and heat transfer models are strongly related to accurate values for transport coefficients. The Navier-Stokes equation [1] and the energy equation [2] incorporate for a given substance three transport coefficients that connect the physical dynamics of momentum and heat to three properties, namely, bulk viscosity, shear viscosity and thermal conductivity.

There are in the literature several data sets that collect experimental values of shear and thermal conductivity for simple substances. The electronic tables from the National Institute of Standards and Technology (NIST) [3], for instance, allow the solution of a great number of typical engineering problems. In these cases, the bulk viscosity is generally neglected based on the validity of Stokes hypothesis [4] even when the fluid is neither incompressible nor a monoatomic dilute gas [5]. Hence, bulk viscosity values are rarely found in tables, technical works and engineering projects, even though they are needed for the complete specification of the Navier-Stokes stress tensor.

\footnotetext{
* Corresponding author at: Defense Engineering Graduate Program, Military Institute of Engineering, 22290-270 Rio de Janeiro, Brazil.

E-mail address: leonardo.alves@mec.uff.br (L.S. Alves).
}

However, in the neighborhood of the critical point, availability of transport coefficient data is scarce. Moreover, theory and experiment display anomalies in the behavior of some thermodynamic and transport properties, usually appears as a divergence towards infinite or zero depending on the property [6-10]. In particular, the high compressibility of near-critical fluids reduces the accuracy of experimental measurements. For this class of fluids, bulk viscosity data are even more limited since the acoustic absorption of sound waves - the most used experimental technique [11,12] - is subject to high levels of error. To overcome this drawback, Brillouin scattering was developed as a promising technique to measure bulk viscosity, though it is still not widely known and used [13].

In order to overcome the aforementioned experimental limitations, an interesting possibility is performing molecular simulations that can suitably model the near-critical state and compute transport coefficients. One of these methods is equilibrium molecular dynamics (MD) [14-16]. In this approach, fundamental thermophysical properties such as energy, temperature, pressure can be determined from specific averages of the dynamic evolution of an ensemble of molecules. Other properties are obtained using relations from thermodynamics and statistical mechanics including time correlations, e.g., transport properties [17]. This method is useful in the investigations of phase diagrams [18] and chemical reactions [19]. In the framework of MD, particle tra- 

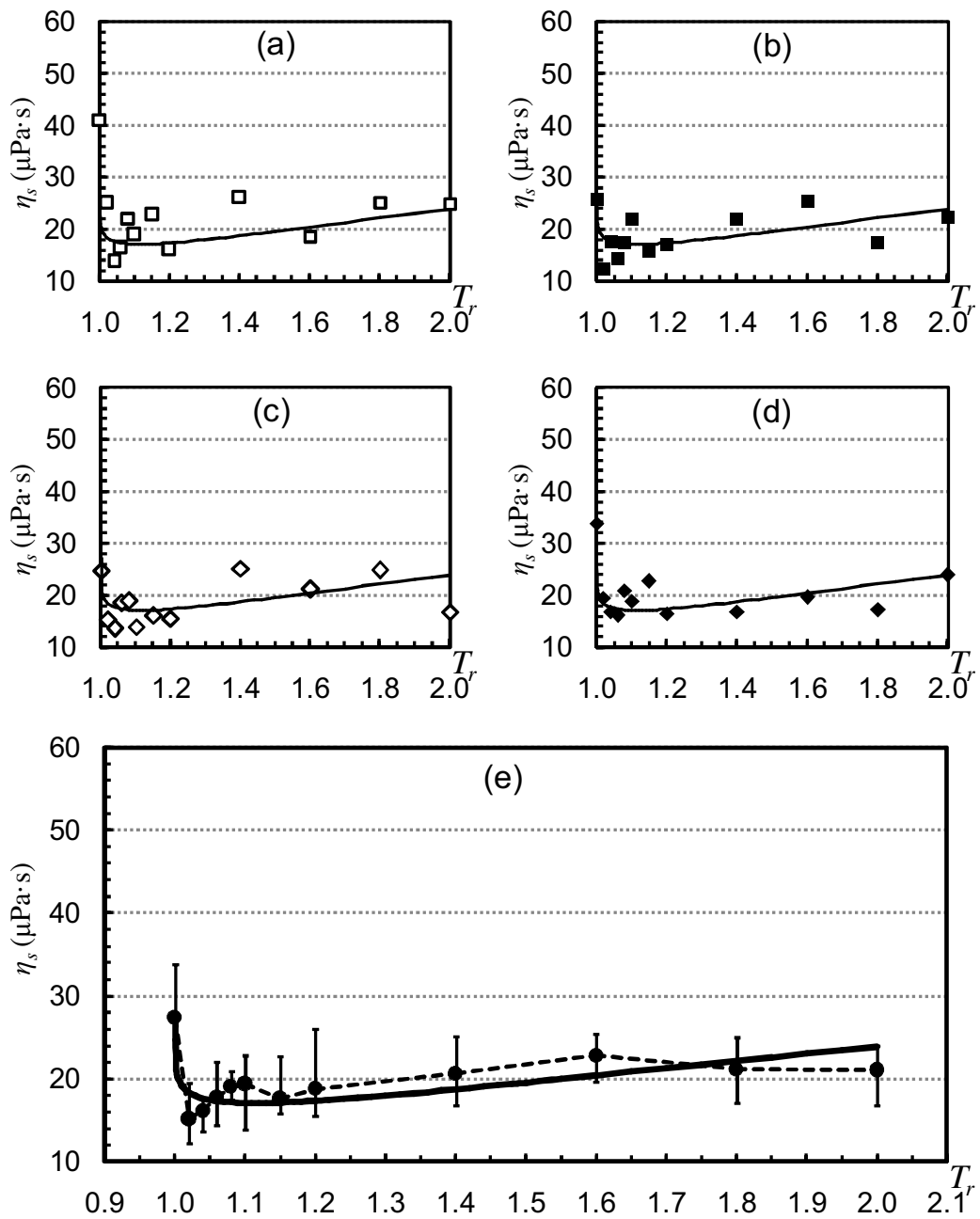

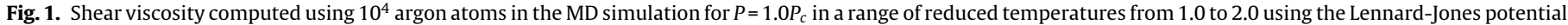
with different parameters pairs - (a) [25], (b) [26], (c) [29], (d) [30] - and (e) the average values with error bars.

jectories in phase space defined by positions and velocities are computed from the forces resulting from an interaction potential energy between pairs of atoms and molecules. The most employed model for non-bonded particles is the Lennard-Jones (L-J) potential [20]. It has a simple mathematical expression and leads to accurate results notably in the study of noble gases. The L-J has already been used to compute transport properties of supercritical fluids far from their critical points, transport properties of near-critical fluids using other approaches and other properties [21-26].

In this work, we investigated transport properties of nearcritical argon using equilibrium MD. The present simulations are based on Green-Kubo theory and use four different versions of argon Lennard-Jones potentials available in the literature. The importance of investigating argon in this work is twofold. First, it is the most abundant noble gas in the atmosphere. Second, it is an important substance employed for calibration purposes in laboratories because it is chemically inert and has a low cost. The present results were compared with available experimental data, which allows one to estimate their range of applicability. Due to the scarcity of bulk viscosity experimental values, especial emphasis was given to this transport property in such a way that they can be used in simulations of compressible fluid flow [27] as well as heat transfer problems [28].

\section{Fundamentals}

\subsection{Molecular dynamics and the Lennard-Jones potential}

The basis of the MD technique is the computational simulation of Newton's equation of motion for a set of interacting moving particles. For a system consisting of argon atoms in a regular box, only interactions between pairs of non-bonded atoms were considered. In other words, many-body interactions are not considered explicitly.

A pair potential suitably models the interactions between atom pairs. The simplest realistic model for this purpose is the L-J 12-6 potential. It is a two parameters model given by Eq. (1)

$U_{\mathrm{LJ}}\left(r_{i j}\right)= \begin{cases}4 \varepsilon\left[\sigma / r_{i j}{ }^{12}-\sigma / r_{i j}{ }^{6}\right], & r_{i j} \leq r_{c} \\ 0, & r_{i j}>r_{c}\end{cases}$

where $\varepsilon$ is the depth of the potential well, $\sigma$ is the zero-energy distance, $r_{i j}$ is the distance between particles $i$ and $j$, and $r_{c}$ is a cut-off distance that plays an important role in reducing the computational cost. To use this potential it is necessary to correct the computed value of the potential in order to include the small energies resulting from long-range interactions farther than $r_{c}$ [20]. The 12 -exponent term models repulsion effects while the 6 -exponent 

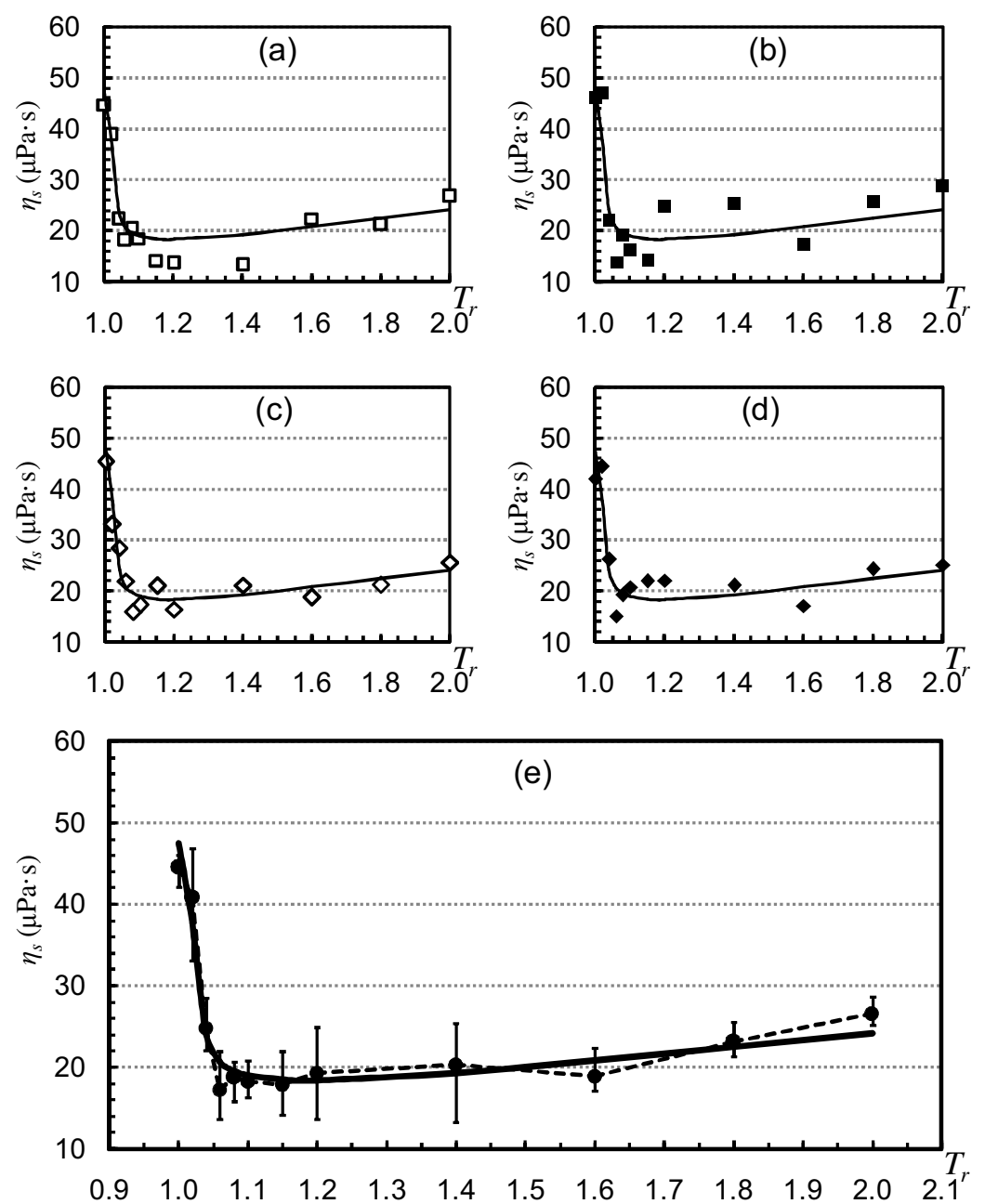

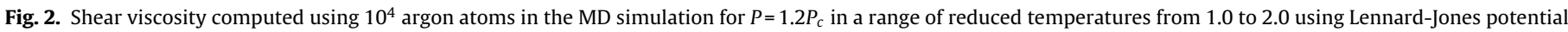
with different parameters pairs - (a) [25], (b) [26], (c) [29], (d) [30] - and (e) the average values with error bars.
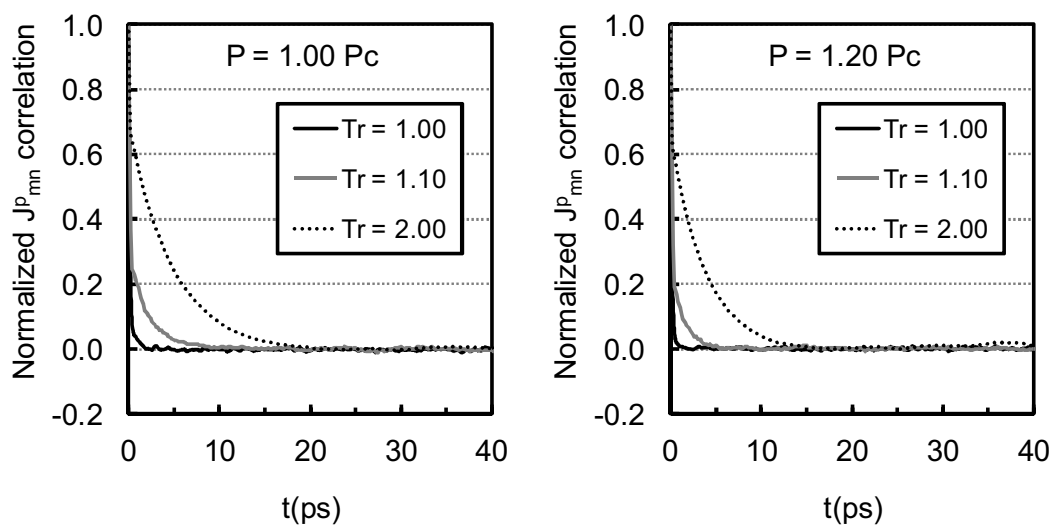

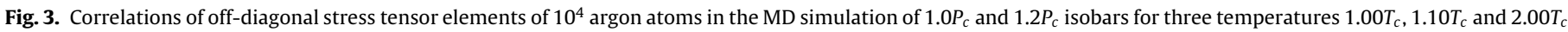
using Lennard-Jones potential parameters from [25].

term describes attraction effects. Each $i-j$ pair of particles interacts via a force $\boldsymbol{f}_{i j}$ that can be calculated from

$\boldsymbol{f}_{i j}=-\nabla U_{\mathrm{LJ}}\left(r_{i j}\right)$

The motion of each particle is determined from its resultant force. An important feature of MD is that an accurate potential energy expression generally leads to accurate thermophysical properties. When using the Lennard-Jones 12-6 expression, all possible model choices are restricted to selecting $\sigma$ and $\varepsilon$ values in addition to a special care concerning the $r_{c}$ values in all cases. In order to consider uncertainties of the LJ potential parametrization of argon, four different pairs of $\sigma$ and $\varepsilon$ and their respective critical constants were used and compared in this work. They are shown in Table 1.

In MD simulations it is possible to adopt the concept of statistical ensemble from statistical mechanics. One can simulate an 

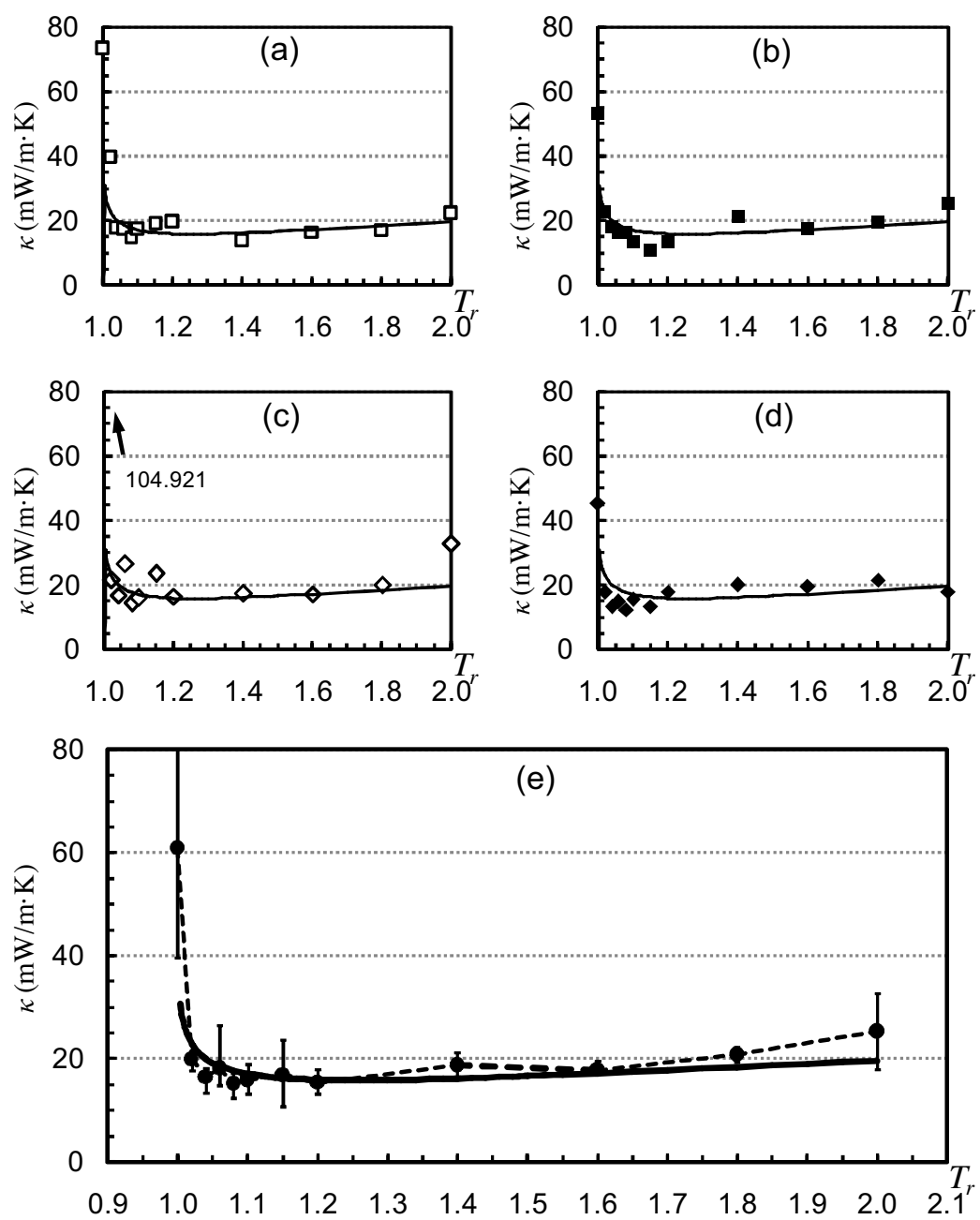

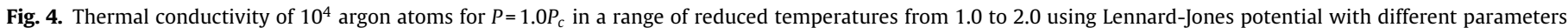
pairs - (a) [25], (b) [26], (c) [29], (d) [30] - and (e) the average values with error bars.

Table 1

Parameter pairs for the Lennard-Jones 12-6 potential argon atoms along with their respective critical constants.

\begin{tabular}{lllllll}
\hline \multicolumn{2}{l}{ Model Parameters } & & \multicolumn{3}{l}{ Critical Constants } & Ref. \\
\cline { 1 - 2 } \cline { 5 - 6 }$\sigma(\AA)$ & $\varepsilon / \mathrm{k}_{\mathrm{B}}\left(\mathrm{K}^{-1}\right)$ & & $\mathrm{T}_{\mathrm{C}}(\mathrm{K})$ & $\mathrm{P}_{\mathrm{c}}(\mathrm{atm})$ & $\rho_{\mathrm{c}}\left(\mathrm{kg} / \mathrm{m}^{3}\right)$ & \\
\hline 3.405 & 119.8 & & 150.86 & 48.34 & 535.397 & Michels et al. [25] \\
3.43 & 119.3 & & 150.86 & - & 535.397 & Levelt [26] \\
3.345 & 125.7 & & 150.86 & - & 527.265 & White [29] \\
3.3952 & 116.79 & & 153.00 & 51.42 & 532.107 & Vrabec et al. [30] \\
\hline
\end{tabular}

$\overline{\mathrm{T}_{\mathrm{c}} \text { : critical temperature; } \mathrm{P}_{\mathrm{c}} \text { : critical pressure; } \rho_{\mathrm{c}} \text { : critical density; } \mathrm{k}_{\mathrm{B}} \text { : Boltzmann }}$ constant.

isolated system (e.g., microcanonical or NVE ensemble), a closed system (canonical, isochoric-isothermal or NVT ensemble), an isobaric-isothermal system (NPT ensemble), among other possibilities, without using partition functions that may be difficult to compute. Before physical arguments are considered, the choice of an ensemble is related to the use of a specific numerical integration scheme of the equations of motion. Each algorithm can include artificial thermostats or barostats to control the basic thermodynamic variables $-N, V, T$ and $P[14]$. The mathematical expressions used in MD and derived from statistical mechanics theory for computing the thermophysical properties of interest must be obtained from suitable ensembles.

It is also important to avoid edge effects. For this purpose, MD simulations of bulk systems generally employ periodic boundary conditions. These artificial boundaries imposed on the system restrict the simulation box size. This approach is called minimal-image convention and it states that the characteristic dimension of the simulation box - the length for a cubic box - must be greater than twice the cutoff radius $r_{c}$.

\subsection{Green-Kubo formulas for transport properties}

Equilibrium MD employs Green-Kubo formulas [31] for the computation of shear and bulk viscosity as well as of thermal conductivity. The three coefficients could be obtained experimentally by perturbing the system towards non-equilibrium states and observing its response that is associated with irreversible processes. Therefore, in principle, to compute them one should use non-equilibrium molecular dynamics (NEMD) instead of equilibrium MD. NEMD is based on the direct application of a heat or momentum flux on the simulation box $[32,33]$. On the other hand, the fluctuation dissipation theorem allows the computation of transport coefficients from the microscopic fluctuations of the system. This theorem states that small fluctuations of the system are related to internal relaxation processes. Therefore, transport coefficients then can be calculated as integrals of time-correlation functions of the microscopic stress tensor and internal energy fluxes in an equilibrium state.

Shear viscosity $\eta_{S}$ is related to the resistance of the fluid to shear forces. In the microcanonical and canonical ensembles this 

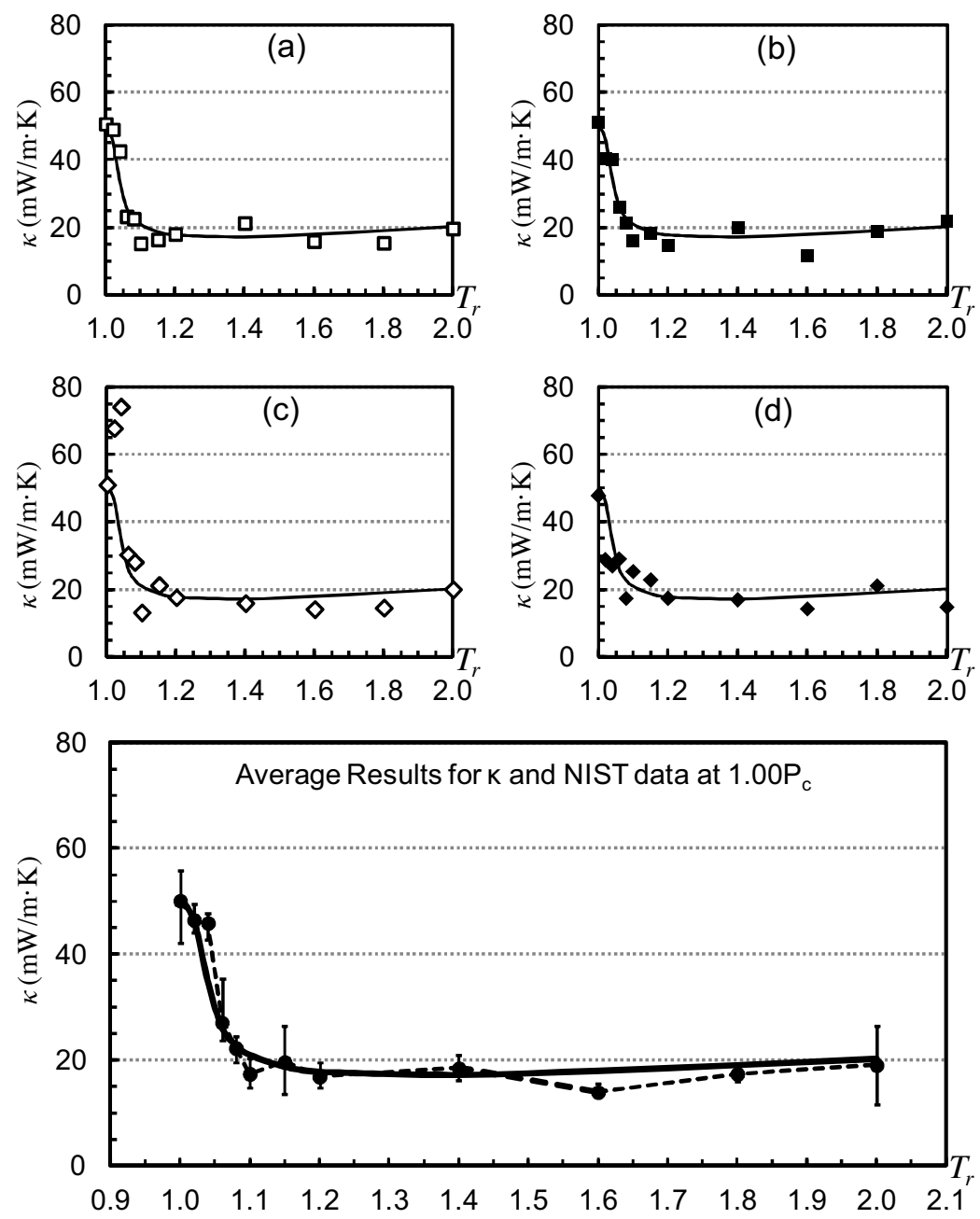

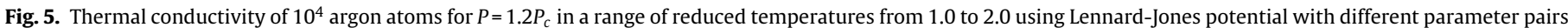
- (a) [25], (b) [26], (c) [29], (d) [30] - and (e) the average values with error bars.
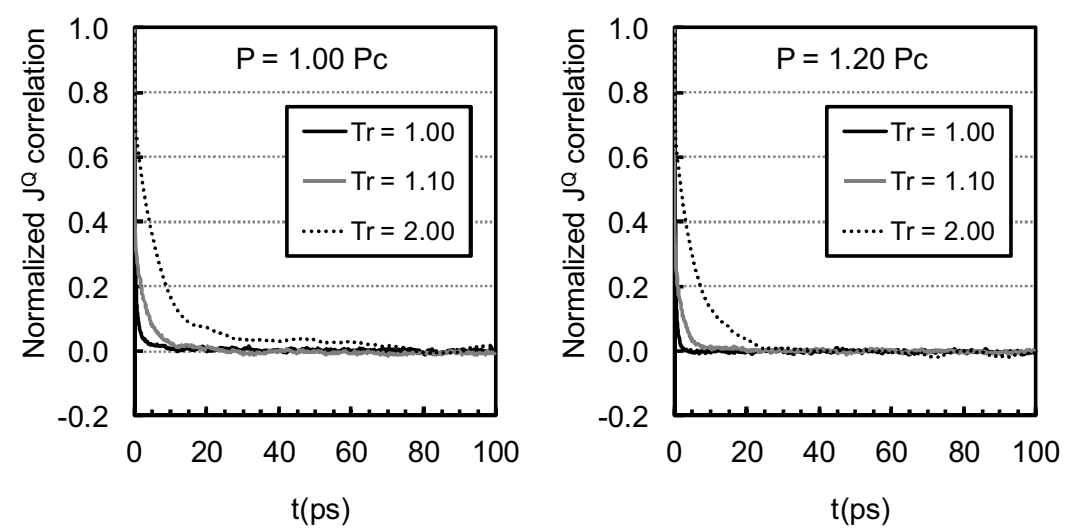

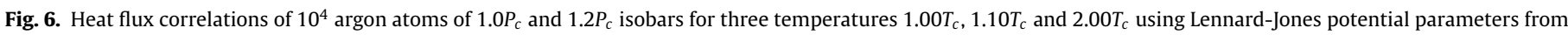
[25].

property is obtained by integrating the ensemble average of the time correlation function of the stress tensor $\boldsymbol{J}^{p}$ off-diagonal elements, which represent the momentum fluxes,

$\eta_{s}=\frac{1}{3 k_{B} V T} \sum_{m<n} \int_{0}^{\infty}\left\langle J_{m n}^{p}(0) J_{m n}^{p}(t)\right\rangle d t$ where $m$ and $n$ are indexes representing the Cartesian coordinates $x, y$ and $z$ with $m \neq n, k_{B}$ is the Boltzmann constant, and $V$ and $T$ are respectively the volume and temperature of the system.

The bulk viscosity $\eta_{b}$ describes the resistance of a fluid to dilatation assuming a small volume element of constant shape. In the microcanonical ensemble this property is obtained by integrating 

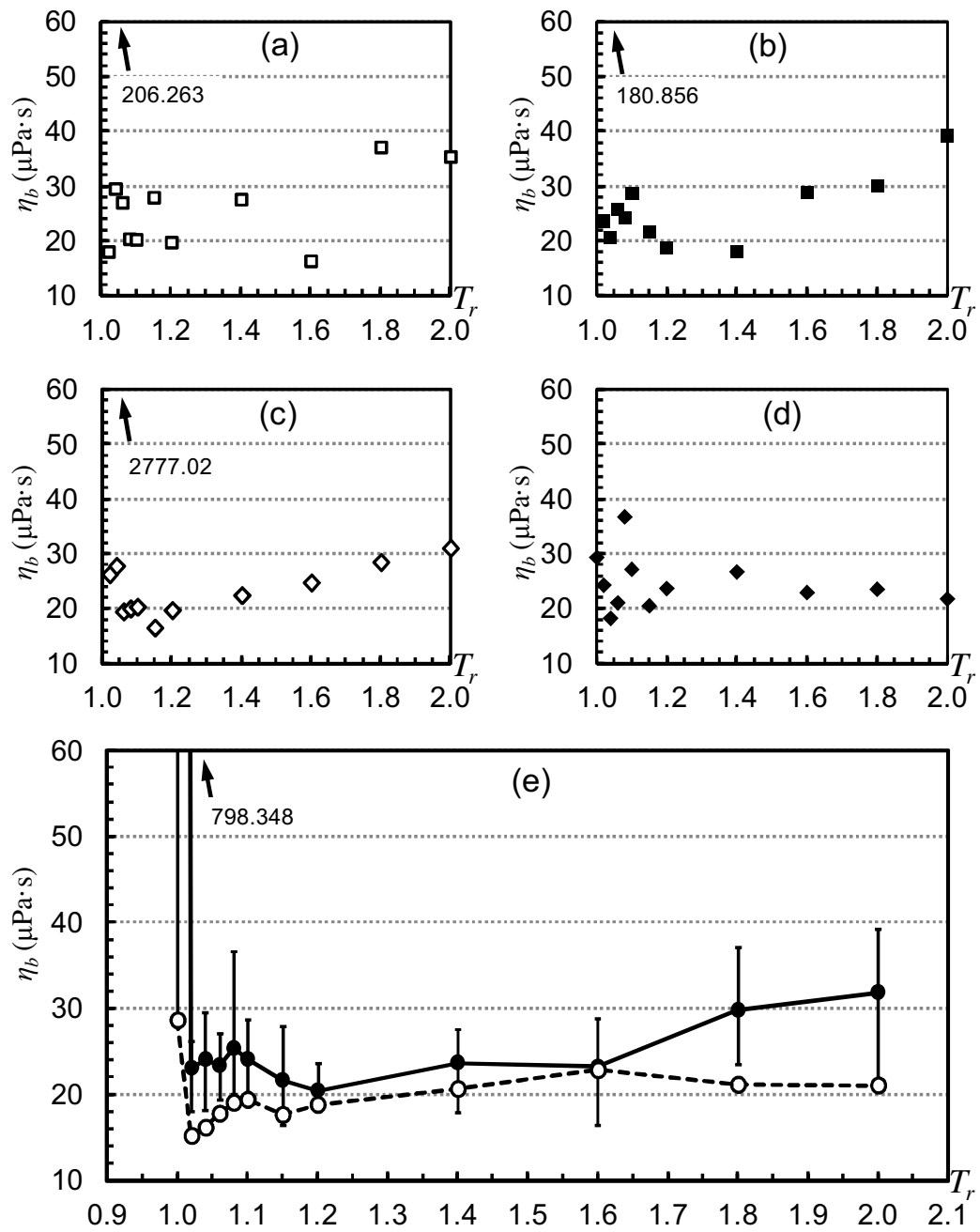

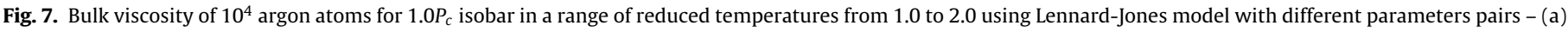
[25], (b) [26], (c) [29], (d) [30] - and (e) the average values of $\eta_{b}$ compared to the average values of $\eta_{s}$ for the same isobar.

the ensemble average of the time correlation function of the stress tensor $\boldsymbol{J}^{p}$ diagonal elements minus the product $P V$. In other words,

$\eta_{b}=\frac{1}{3 k_{B} V T} \sum_{m} \int_{0}^{\infty}\left\langle\left(J_{m m}^{p}(0)-P(0) V\right)\left(J_{m m}^{p}(t)-P(t) V\right)\right\rangle d t$

Thermal conductivity, $\kappa$, physically expresses the abilityof the substance in transferring thermal energy when subject to temperature gradients. It is computed by integrating the ensemble average of time correlation function of the microscopic heat flux $\boldsymbol{J}^{Q}$ vector, namely,

$\kappa=\frac{1}{3 k_{B} V T^{2}} \int_{0}^{\infty}\left\langle\boldsymbol{J}^{Q}(0) \cdot \boldsymbol{J}^{Q}(t)\right\rangle d t$

In Eqs. (3) and (4), the stress tensor $J^{\mathrm{P}}$ can be computed from

$\boldsymbol{J}^{p}=\sum_{i=1}^{N} m_{i} \boldsymbol{v}_{i} \boldsymbol{v}_{i}^{\prime}-\sum_{i=1}^{N} \sum_{j>i}^{N} \boldsymbol{r}_{i j}-\left(\nabla U_{i j}\right)^{\prime}$

where $m_{i}$ is the mass of the particle $i, U_{i j}$ the interaction potential between particles $i$ and $j$, and the prime means matrix transpose. Diagonal elements have the index $m$ equal to $n$. In Eq. (5), the energy flux vector is calculated from

$\boldsymbol{J}^{Q}=\frac{1}{V}\left[\sum_{i=1}^{N} e_{i} \boldsymbol{v}_{i}+\frac{1}{2} \sum_{i<j 1}^{N}\left(-\nabla U_{i j} \cdot\left(\boldsymbol{v}_{i}+\boldsymbol{v}_{j}\right)\right) \boldsymbol{r}_{i j}\right]$

where $e_{i}$ is the energy, potential and kinetic, of particle $i$ whereas $v_{i}$ is the velocity of particle $i$.

\subsection{Near-critical effects}

The thermophysical and rheological properties of a substance in the neighborhood of the critical point are characterized by an anomalous behavior. These critical anomalies are observed as divergences either to zero or infinity. Theory and experiment show that bulk and shear viscosities and thermal conductivity diverge to infinity in the vicinity of the critical point $[34,23,35-39,21,40,41]$. One important feature of these divergences is their universality, i.e., they are described by universal parameters and expressions that do not depend on the nature of the fluid. These models are power laws having a single parameter that indicates the separation of each thermodynamic state from the critical point. For the two viscosities and thermal conductivity, this parameter is the temperature difference $\Delta T=T-T_{c}$. However, it is also important to identify the correct thermodynamic path of the power law model, since 

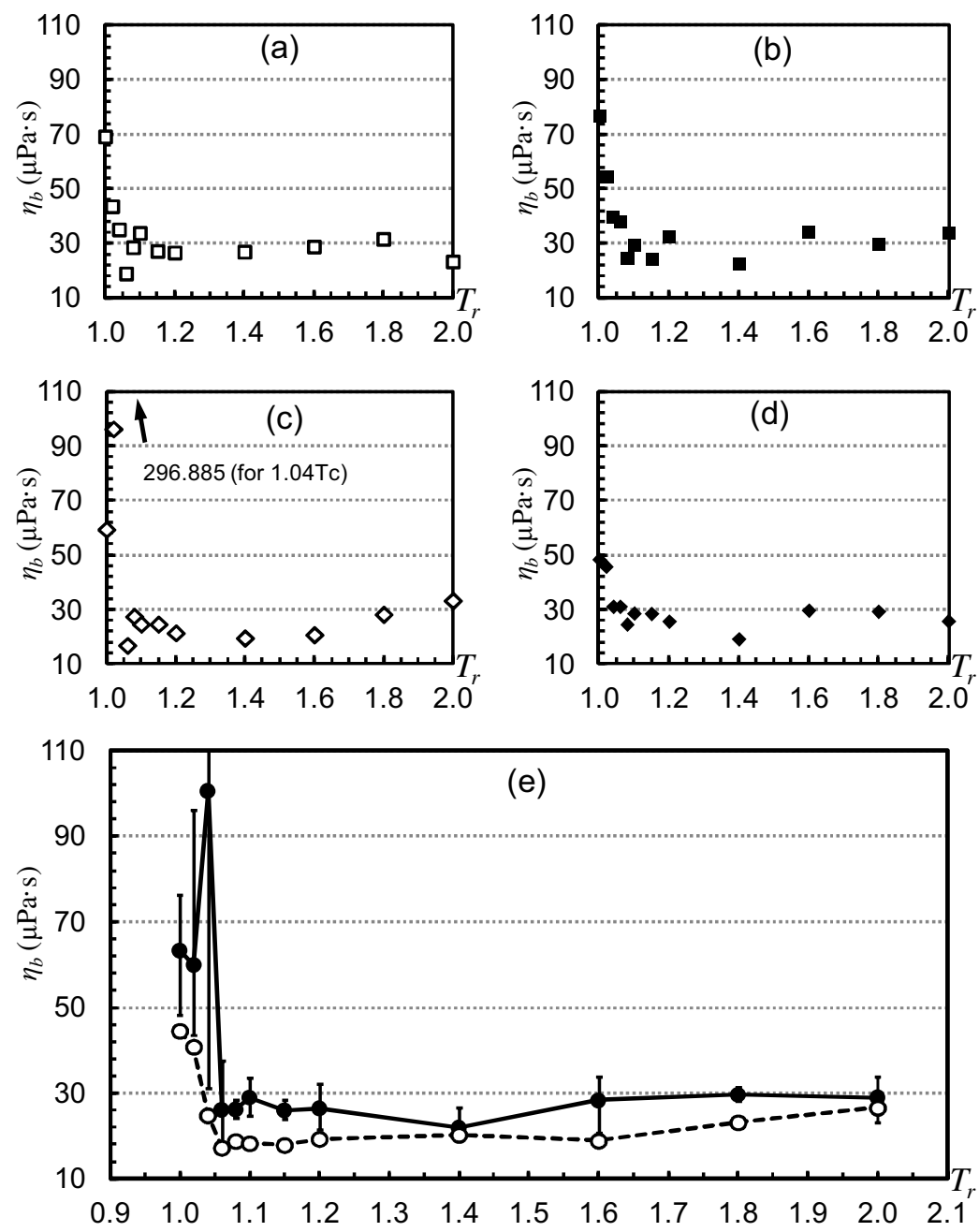

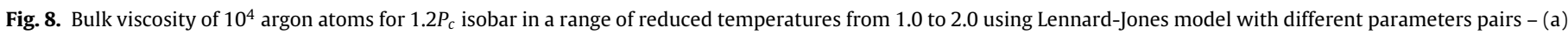
[25], (b) [26], (c) [29], (d) [30] - and (e) the average values of $\eta_{b}$ compared to the average values of $\eta_{s}$ for the same isobar.
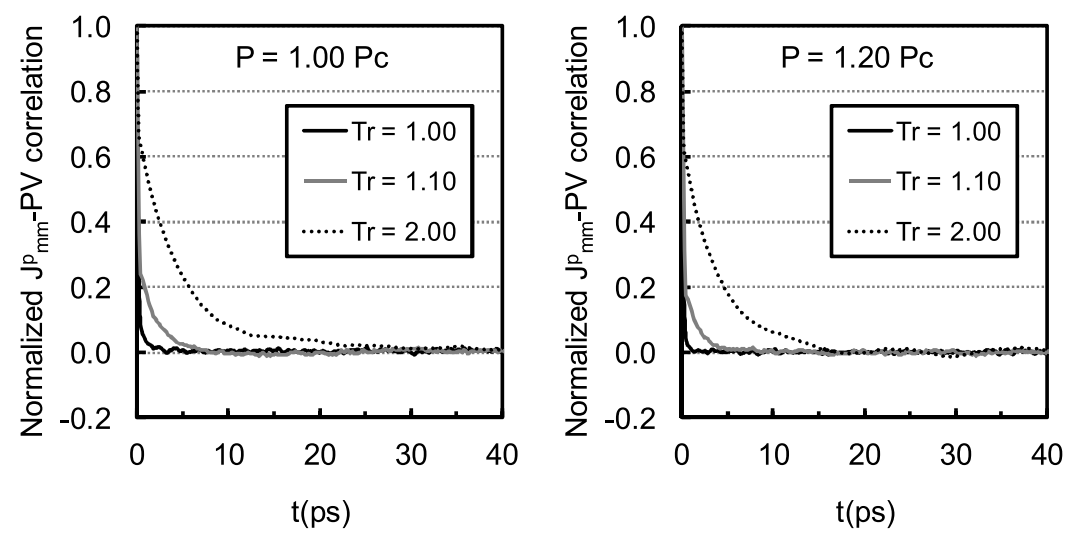

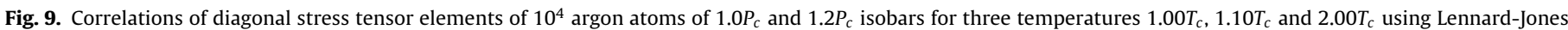
potential parameters from [26].

different paths lead to different power law coefficients that could be related through an equation of state [6]. For instance, along the critical isochore, one has

$\kappa \approx \kappa^{\circ} \Delta T^{\nu+\eta-\gamma}$

$\eta_{s} \approx \eta_{s}^{\circ} \Delta T^{-\eta}$ where $v=0.630 \pm 0.001, \eta=0.031 \pm 0.004$ and $\gamma=1.239 \pm 0.002$ are universal exponents obtained from renormalization group theory [42], with $\kappa^{\circ}$ and $\eta_{s}^{\circ}$ depending on the substance. In particular, studies seeking to understand the behavior of bulk viscosity near critical conditions are rare. Five of the most representative ones based either on Monte Carlo simulations, or on MD and even purely theoretical $[21,43-46]$ did not describe the divergence of 
this property in terms of the classical critical exponents. Furthermore, scaling laws similar to the ones given by Eqs. (8) and (9) were not found along isobars. Therefore, the present work is especially important since it can help overcoming these difficulties as shown next.

\section{Computational methods}

The MD simulations were carried out usingthe open source code LAMMPS [47]. Shear viscosity and thermal conductivity were computed in the NVT ensemble using a Nosé-Hoover style nonHamiltonian equation of motion [48] in order to improve the control of the simulation temperature. In order to prevent any influence over pressure fluctuations, bulk viscosity values were obtained in the NVE ensemble using the Verlet algorithm.

Following recent papers focusing on near critical MD simulations $[9,49]$, we used $10^{4}$ argon atoms in all simulations. This number of particles leads to a better control of the thermodynamic state in the simulation box and improved statistical averages. The numerical integration of the time correlation functions of the transport coefficients (Eqs. (3)-(5)) used the trapezoidal rule over all time steps. Each simulation was equilibrated for $2 \mathrm{~ns}\left(2 \times 10^{6}\right.$ time steps) before averages were computed for a time span of $10 \mathrm{~ns}\left(10^{7}\right.$ time steps). A timestep of $1 \mathrm{fs}$ was used. Correlations of heat and momentum fluxes were computed for a sampling length of $200 \mathrm{ps}$ $\left(2 \times 10^{5}\right.$ time steps). A large cut off radius of $8 \sigma$ was chosen to improve the accuracy in the calculation of the interaction energies and better describe long-range correlations typical of the critical region. The correction in the potential for $r_{i j}>r_{c}$ was computed integrating a unitary radial distribution function for these distances [20]. By employing a large value of the $r_{c}$ distance, simulations were more realistic for a dense fluid and described more accurately long-range corrections. Periodic boundary conditions were used. A temperature range from $1.0 T_{c}$ up to $2.0 T_{c}$ along two isobars $-1.0 P_{c}$ and $1.2 P_{c}$ - was considered. The four pairs of L-J parameters shown in Table 1 were used in the computations. More details about the simulation procedure can be found elsewhere [18].

\section{Results and discussion}

Figs. 1 and 2 show shear viscosity results of argon obtained from the MD simulations along the $1.0 P_{c}$ and $1.2 P_{c}$ isobars using four different sets of L-J parameters taken from the literature (Table 1).

There are no significant differences between the results computed using all the L-J parameter pairs according to Table 1. Therefore, computation of the shear viscosity is barely sensitive to small variations in the parameters of the interaction potential. Furthermore, MD simulations were able to reproduce the behavior of the shear viscosity critical divergence quite well. Figs.1e and 2e show that simulations are in good agreement with NIST data. The results obtained using Michels et al. [25] parameters for the L-J potential exhibit a better description of the critical divergence in the shear viscosity.

To ensure the convergence of the results in Figs. 1 and 2, we present in Fig. 3 a set of normalized $\boldsymbol{J}_{m n}$ correlation functions of three representative temperatures for both isobars. The results agree with data already reported in literature [50].

Figs. 4-6 show the computed thermal conductivity values and the heat flux correlation functions of argon, as done for the shear viscosity. The results are also in good agreement with the NIST data. All parameters pairs lead to similar results with small discrepancies as compared to the average values. The parameter pair from Michels et al. [25] displays a smoother behavior in comparison to NIST data and better represented the critical divergence. The error bars are smaller as compared to the shear viscosity ones. In Fig. 6, overall the heat flux correlation function converges more slowly than the respective shear stress correlation in agreement with previously reported information [51]. It is important to point out that this plot shows correlations up to 100 ps and the simulations considered the correlations up to $200 \mathrm{ps}$.

Finally, Figs. 7-9 collects MD bulk viscosity results for argon and the set of correlation functions used to compute them. Since these data are not available in NIST tables, they can be especially useful. Once again, all four pairs of L-J parameters lead to similar results, but in this case the L-J parameters from White [29] showed a clearly smoother behavior and a better description of the critical divergence. Large deviations were found in the average value of bulk viscosity results. These deviations are considerably larger than deviations reported for shear viscosity and thermal conductivity. Moreover, Fig. 9 shows that the diagonal stress tensor correlations exhibit a better convergence if compared to thermal conductivity correlations. The correlation analysis of the bulk viscosity suggests that this property is easier to compute as compared to thermal conductivity even near the critical point.

Another important feature in Figs. 7 and 8 is the non-zero value of the bulk viscosity far from the critical temperature. At $T_{r}=2.0$ $(\approx 300 \mathrm{~K})$ this property reaches values around $30 \mu \mathrm{Pa}$ s with an average deviation of $\pm 5 \mu$ Pa s considering the results obtained from all set of L-J parameters. This result shows that even though argon is a monoatomic gas, the Stokes hypothesis as well as the kinetic gas theory assumptions [52] do not hold and thus cannot be used. Furthermore, this property has the same order of magnitude of the shear viscosity values and, hence, cannot be neglected in simulations of the momentum conservation equations.

Figs. 7e and 8e compare both viscosities obtained using averages over the MD results and the four sets of L-J parameters at each $T_{r}$ value. These results indicate that bulk viscosity values are actually larger than their shear counterparts for the thermodynamic states considered here. This is an important finding because classical fluid mechanics analysis neglects the bulk viscosity [53]. From these figures we propose an empirical relation between both viscosities at each isobar: one should use $\eta_{b} \approx 1.3 \eta_{s}$ at the $1.0 P_{c}$ isobar and $\eta_{b} \approx 1.6 \eta_{s}$ at the $1.2 P_{c}$ isobar. Therefore, the traditional assumption of neglecting this difference cannot be a priori assumed in the neighborhood of the critical point.

\section{Conclusions}

In this work it was shown that molecular dynamics simulations using the Lennard-Jones potential to describe the potential energy between argon atoms allow one to obtain accurate values of both shear viscosity and thermal conductivity along isobars in the neighborhood of the critical point. The computed properties were in good agreement with experimental data collected in the NIST database. This good agreement leads us to compute also the bulk viscosity in the same thermodynamic region even though NIST data is not available in this case.

In the neighborhood of the critical point, the results for bulk viscosity obtained with molecular dynamics show that: (a) shear and bulk viscosities diverge along the same isobar with similar behavior; (b) bulk viscosity does not go to zero away from the critical point in spite of being close to it, thus contradicting Stokes hypothesis and kinetic theory even for a monoatomic dilute gas; (c) bulk viscosity is not only of the same order of magnitude as shear viscosity near the critical point, but it is actually larger than its counterpart in this region.

The present approach of using molecular dynamics to compute diffusion properties of a fluid in the neighborhood of the critical point is general and we have shown that can be used to obtain reliable results in this experimentally difficult to measure 
thermodynamic region. The crucial point in the method is the choice of the interaction potential, which can be an issue for more complicated and practical systems (e.g., $\mathrm{N}_{2}$ ). Investigations along these lines are under way in our group.

The complete set of results for the transport properties is available as an excel file in the Supplementary material of this work.

\section{Acknowledgements}

L.S. de B.A. thanks Brazilian agencies FAPERJ (through grants E26/110.846/2011 and E-26/111.670/2012), CNPq (through Grant MCT/CNPq14-481072/2012-8) and Capes. I.B. and J.N. thank the same agencies for support of this research as well the Brazilian Army. L.S. de B.A. acknowledges the financial support from the United States Air Force (through the SOARD grant FA9550-13-10178). A.B.O. thanks CNPq (through Grants 303820/2013-6 and 459852/2014-0) as well as partial financial support from PROPPUFOP (through Auxílio a Pesquisadores 2015 Grant). We would like also to thank the referees for the valuable suggestions concerning data analysis.

\section{Appendix A. Supplementary data}

Supplementary data associated with this article can be found, in the online version, at http://dx.doi.org/10.1016/j.supflu.2016.04. 004.

\section{References}

[1] P.K. Kundu, I.M. Cohen, D.R. Dowling, Fluid Mechanics, 6th ed., Academic Press, London, 2015.

[2] D.W. Hahn, M.N. Özisik, Heat Conduction, 3rd ed., John Wiley \& Sons, New York, 2012

[3] National Institute of Standards and Technology Thermophysical Properties of Fluid Systems, 2011 (Available:) http://webbook.nist.gov/chemistry/fluid/.

[4] G.G. Stokes, On the theories of internal friction of fluids in motion, Trans. Cambridge Philos. Soc. 8 (1845) 287-305.

[5] M. Gad-el-Hak, Stoke's hypothesis for a newtonian, isotropic fluid, J. Fluids Eng. 117 (1995) 3-5.

[6] P. Carlès, A brief review of the thermophysical properties of supercritical fluids, J. Supercrit. Fluids 53 (2012) 2-11.

[7] D. Beysens, J. Straub, D. Turner, Phase transitions and near-critical phenomena, in: H.U. Walter (Ed.), Fluid Sciences and Materials Science in Space, Springer-Verlag, Berlin, 1987, pp. 221-256.

[8] H.E. Stanley, Introduction to Phase Transitions and Critical Phenomena, Oxford University Press, Oxford, 1971.

[9] D. Bolmatov, V.V. Brazhkin, K. Trachenko, Thermodynamic behaviour of supercritical matter, Nat. Commun. 4 (2013) 2331.

[10] T.M. Yigzawe, R.J. Sadus, Intermolecular interactions and the thermodynamic properties of supercritical fluids, J. Chem. Phys. 138 (2013) 194502.

[11] R.E. Graves, B.M. Argrow, Bulk viscosity: past to present, J. Thermophys. Heat Transf. 13 (1999) 337-342.

[12] J.A. Cowan, P.W. Ward, Ultrasonic attenuation and the bulk viscosity of liquid argon near the critical point, Can. J. Phys. 51 (1973) 2219-2224.

[13] X. He, H. Wei, J. Shi, J. Liu, S. Li, W. Chen, X. Mo, Experimental measurement of bulk viscosity of water based on stimulated Brillouin scattering, Opt. Commun. 285 (2012) 4120-4124.

[14] S.B. Frenkel, B. Smit, Understanding Molecular Simulation, 2nd ed., Academic Press, San Diego, 2002.

[15] K. Binder, Computer simulations of critical phenomena and phase behaviour of fluids, Mol. Phys. 108 (2010) 1797-1815.

[16] P.T. Cummings, Molecular simulation of near-critical and supercritical fluids, in: E. Kiran, J.M.H. Levelt-Sengers (Eds.), Supercritical Fluids: Fundamentals and Applications, Kluwer Academic Publishers, Dordrecht, 1994, pp. 387-409.

[17] D.A. McQuarrie, Statistical Mechanics, University Science Books, Mill Valley, 2000.

[18] J. Nichele, L.S. de B. Alves, I. Borges Jr., Equation of State for near critical argon obtained via molecular dynamics, High Temp.-High Pressures 43 (2014) 385-400.

[19] E. Martínez-Núñez, I. Borges Jr., S.A. Vázquez, Association rate constants for the $\mathrm{CH}_{3}+\mathrm{NO} \rightarrow \mathrm{CH}_{3} \mathrm{NO}$ reaction by classical trajectory and CVTST calculations, J. Phys. Org. Chem. 15 (2002) 123-129.
[20] M.P. Allen, D.J. Tildesley, Computer Simulation of Liquids, Oxford University Press, Oxford, 1987.

[21] S. Roy, S.K. Das, Transport phenomena in fluids: finite-size scaling for critical behavior, Europhys. Lett. 94 (2011) 36001.

[22] T. Hamanaka, R. Yamamoto, A. Onuki, Molecular dynamics simulation of heat conduction in near-critical fluids, Phys. Rev. E 71 (2005) 011507.

[23] O.C. Nwobi, L.N. Long, M.M. Micci, Molecular dynamics studies of transport properties of supercritical fluids, in: Proceedings of the 35th AIAA Aerospace Sciences Meeting \& Exhibit, Reno-NV, 1997, pp. 1-11.

[24] R.L. Rowley, M.M. Painter, Diffusion and viscosity equations of state for a Lennard-Jones fluid obtained from molecular dynamics simulations, Int. J. Thermophys. 18 (1997) 1109-1121.

[25] A. Michels, H. Wijker, H.K. Wijker, Isotherms of argon between $0^{\circ} \mathrm{C}$ and $150^{\circ} \mathrm{C}$ and pressures up to 2900 atmospheres, Physica 15 (1949) 627-633.

[26] J.H.M. Levelt, The reduced equation of state, internal energy and entropy of argon and xenon, Physica 26 (1960) 361-377.

[27] P.C. Teixeira, L.S. de B. Alves, Piston effect characteristic time dependence on equation of state model choice, in: Proceedings of CHT-12, ICHMT Digital Library, Bath, 2012.

[28] L.S. de B. Alves, An integral transform solution for unsteady compressible heat transfer in fluids near their thermodynamic critical point, Therm. Sci. 17 (2013) 673-686

[29] J.A. White, Lennard-Jones as a model for argon and test of extended renormalization group calculations, J. Chem. Phys. 111 (1999) 9352-9356.

[30] J. Vrabec, J. Stoll, H. Hasse, A set of molecular models for symmetric quadrupolar fluids, J. Phys. Chem. B 105 (2001) 12126-12133.

[31] R. Zwanzig, Time-correlation functions and transport coefficients in statistica mechanics, Annu. Rev. Phys. Chem. 16 (1965) 67-102.

[32] G. Galliéro, C. Boned, A. Baylaucq, Molecular dynamics study of the Lennard-Jones fluid viscosity: application to real fluids, Ind. Eng. Chem. Res. 44 (2005) 6963-6972.

[33] D.J. Evans, Thermal conductivity of the Lennard-Jones fluid, Phys. Rev. A 34 (1986) 1449-1453.

[34] B.J. Bailey, K. Kellner, The thermal conductivity of argon near the critical point, Br. J. Appl. Phys. 18 (1967) 1645-1647.

[35] H.J.M. Hanley, R.D. McCarty, J.V. Sengers, Viscosity and thermal conductivity coefficients of gaseous and liquid oxygen, in: NASA Cr-2440, University of Maryland, 1974.

[36] B.A. Younglove, H.J.M. Hanley, The viscosity and thermal conductivity coefficients of gaseous and liquid argon, J. Phys. Chem. Ref. Data 15 (1986) 1323-1337.

[37] K. Meier, Computer Simulation and Interpretation of the Transport Coefficients of the Lennard-Jones Model Fluid, University of the Federal Armed Forces Hamburg, Hamburg, 2002 (Thesis).

[38] L.P. Kadanoff, J. Swift, Transport coefficients near the liquid-Gas critical point, Phys. Rev. 166 (1968) 89-101.

[39] B.W. Tiesinga, E.P. Sakonidou, H.R. van der Berg, The thermal conductivity of argon in the critical region, J. Chem. Phys. 101 (1994) 6944-6963.

[40] A.E. Nasrabad, R. Laghaei, B.C. Eu, Molecular theory of thermal conductivity of the Lennard-Jones fluid, J. Chem. Phys. 124 (2006) 084506.

[41] R. Vogelsand, C. Hoheisel, Thermal conductivity and shear viscosity of liquid and fluid argon. An extended comparison between experimental and molecular dynamics data, Phys. Chem. Liq. 18 (1988) 141-149.

[42] J.V. Sengers, J.G. Shanks, Experimental critical-exponent values for fluids, J. Stat. Phys. 137 (2009) 857-877.

[43] K. Meier, A. Laesecke, S. Kabelac, Transport coefficients of the Lennard-Jones model fluid. III. Bulk viscosity, J. Chem. Phys. 122 (2005) 014513.

[44] A. Onuki, Bulk viscosity near the critical point, J. Phys. Soc. Japan 66 (1997) 511-513.

[45] G.A. Fernández, J. Vrabec, H. Hasse, A molecular simulation study of shear and bulk viscosity and thermal conductivity of simple real fluids, Fluid Phase Equilib. 221 (2004) 157-163.

[46] M.S. Zabaloy, V.R. Vasquez, E.A. Macedo, Viscosity of pure supercritical fluids, J. Supercrit. Fluids 36 (2005) 106-117.

[47] S.J. Plimpton, Fast parallel algorithms for short-range molecular dynamics, J Comput. Phys. 117 (1995) 1-19.

[48] W. Shinoda, M. Shiga, M. Mikami, Rapid estimation of elastic constants by molecular dynamics simulation under constant stress, Phys. Rev. B 69 (2004) 134103.

[49] V.V. Brazhkin, Y.D. Fomin, A.G. Lyapin, V.N. Ryzhov, E.N. Tsiok, Widom line for the liquid-Gas transition in Lennard-Jones system, J. Phys. Chem. B 115 (2011) $14112-14115$.

[50] K. Meier, A. Laesecke, S. Kabelac, Transport coefficients of the Lennard-Jones model fluid. I. Viscosity, J. Chem. Phys. 121 (2004) 3671-3687.

[51] F. Müller-Plathe, A simple nonequilibiurm molecular dynamics method for calculating the thermal conductivity, J. Chem. Phys. 106 (1997) 6082-6085

[52] W.G. Vincenti, C.H. Kruger, Introduction to Physical Gas Dynamics, Krieger, Malabar-FL, 1965, pp. pp. 407-412.

[53] R.B. Bird, W.E. Stewart, E.N. Lightfoot, Transport Phenomena, 2nd ed., John Wiley \& Sons, New York, 2002. 